\title{
Quantitative Prediction of Individual Psychopathology in Trauma Survivors Using Resting-State fMRI
}

\author{
Qiyong Gong*,', Lingjiang $\mathrm{Li}^{2}$, Mingying Du', William Pettersson-Yeo ${ }^{3}$, Nicolas Crossley $^{3}$, \\ Xun Yang ${ }^{1,4}$, Jing $\mathrm{Li}^{4}$, Xiaoqi Huang ${ }^{1}$ and Andrea Mechelli ${ }^{3}$ \\ 'Huaxi MR Research Center (HMRRC), Department of Radiology, West China Hospital of Sichuan University, Chengdu, China; ${ }^{2}$ Mental Health \\ Institute, The Second Xiangya Hospital of Central South University, Changsha, China; ${ }^{3}$ Department of Psychosis Studies, Institute of Psychiatry, \\ King's College London, London, UK; ${ }^{4}$ Department of Psychiatry, State Key Laboratory of Biotherapy, West China Hospital of Sichuan University, \\ Chengdu, China
}

\begin{abstract}
Neuroimaging techniques hold the promise that they may one day aid the clinical assessment of individual psychiatric patients. However, the vast majority of studies published so far have been based on average differences between groups. This study employed a multivariate approach to examine the potential of resting-state functional magnetic resonance imaging (MRI) data for making accurate predictions about psychopathology in survivors of the 2008 Sichuan earthquake at an individual level. Resting-state functional MRI data was acquired for 121 survivors of the 2008 Sichuan earthquake each of whom was assessed for symptoms of post-traumatic stress disorder (PTSD) using the 17-item PTSD Checklist (PCL). Using a multivariate analytical method known as relevance vector regression (RVR), we examined the relationship between resting-state functional MRI data and symptom scores. We found that the use of RVR allowed quantitative prediction of clinical scores with statistically significant accuracy (correlation $=0.32, P=0.006$; mean squared error $=176.88$, $P=0.00 \mathrm{I}$ ). Accurate prediction was based on functional activation in a number of prefrontal, parietal, and occipital regions. This is the first evidence that neuroimaging techniques may inform the clinical assessment of trauma-exposed individuals by providing an accurate and objective quantitative estimation of psychopathology. Furthermore, the significant contribution of parietal and occipital regions to such estimation challenges the traditional view of PTSD as a disorder specific to the fronto-limbic network.

Neuropsychopharmacology (2014) 39, 68I-687; doi:I0.1038/npp.20I3.25 I; published online 30 October 2013
\end{abstract}

Keywords: trauma; prediction; post-traumatic stress disorder; resting-state; functional magnetic resonance imaging; relevance vector regression.

\section{INTRODUCTION}

Trauma survivors are at high risk of developing symptoms of post-traumatic stress disorder (PTSD), including re-experience of the traumatic event, avoidance of trauma-related stimuli, and hypervigilance (Yehuda and Flory, 2007). However, accurate identification of these symptoms can be difficult due to reliance on a patient's account (Rosen and Taylor, 2007) and high rates of comorbidity with other Axis I psychiatric disorders, somatic disorders, and mild cognitive impairment (Pace and Heim, 2011). Neuroimaging techniques hold the promise that they may one day aid the clinical assessment of individual psychiatric patients, particularly in those cases in which severity of symptoms is difficult to ascertain (Savitz et al, 2013). Consistent with this notion, studies using structural and functional magnetic resonance imaging (MRI) have identified volumetric reductions and

\footnotetext{
* Correspondence: Professor Q Gong, Huaxi MR Research Center, Department of Radiology, West China Hospital of Sichuan University, Chengdu 61004I, China, Tel: + 86-28-85423503,

Fax: + 86-28-85423503 E-mail: qiyonggong@hmrrc.org.cn

Received 7 June 2013; revised 12 August 2013; accepted 29 August

2013; accepted article preview online 25 September 2013
}

functional alternations in trauma-survivors who develop symptoms of PTSD, relative to those who do not, within a distributed fronto-limbic network that includes the dorsolateral and ventromedial prefrontal cortices, amygdalae, and hippocampi (Gilbertson et al, 2002; Pitman et al, 2012; Nardo et al, 2010; Robinson and Shergill, 2011; Shin et al, 2006; New et al, 2009; Lyoo et al, 2011; Morey et al, 2012; Weber et al, 2013). Among these, a number of recent studies using resting-state fMRI specifically have also reported altered functional connectivity in those with PTSD relative to healthy controls, which in turn has been observed to correlate with clinical score (Zhou et al, 2012; Yin et al, 2012; Qin et al, 2012). These differences, however, were based on average estimates at a group level, which is of little use in clinical practice where doctors need to make decisions about individuals. At present, it remains unclear whether neuroimaging could be used to inform the clinical assessment of individual patients.

In an effort to increase the translational applicability of the results, there has been a recent shift toward the use of multivariate machine learning techniques (Brammer, 2009). This alternative approach has two main advantages compared with standard mass-univariate analytical methods that are typically used in neuroimaging. First, it allows 
inferences at the level of the individual and therefore provides results that have higher translational applicability in everyday clinical practice. Second, it is sensitive to differences that are subtle and spatially distributed by virtue of taking inter-regional correlations into account; as such, it provides an ideal framework for investigating psychiatric disorders which affect a distributed network of regions. Here we used a multivariate machine learning technique known as relevance vector regression (RVR) (Tipping, 2001), to evaluate the potential of resting-state functional MRI for making accurate predictions about psychopathology in individuals at high risk of developing PTSD following exposure to trauma. The key strength of RVR relative to other multivariate machine learning techniques such as Support Vector Machine (SVM) (Orrù et al, 2012) is that it allows the quantitative prediction of a variable of interest (eg, a patient's score on a clinical scale) without the need for a discrete categorical decision (eg, patients $v s$ controls). We hypothesized that the application of RVR to resting-state functional MRI data (Zhou et al, 2012; Yin et al, 2012; Qin et al, 2012) would allow accurate quantitative prediction of psychopathology in trauma-survivors. In addition, based on previous studies of the neurobiological basis of PTSD (Gilbertson et al, 2002; Pitman et al, 2012; Nardo et al, 2010; Robinson and Shergill, 2011; Shin et al, 2006; New et al, 2009; Lyoo et al, 2011; Morey et al, 2012; Weber et al, 2013), we expected that accurate quantitative prediction of psychopathology would be informed by functional alterations within a highly distributed network of regions that included the dorsolateral and ventromedial prefrontal cortices, amygdalae, and hippocampi.

\section{MATERIALS AND METHODS}

\section{Subjects}

We recruited 121 survivors of the 2008 Sichuan earthquake between 10 and 15 months after the event (see Table 1). Inclusion criteria included: (i) physically experiencing the earthquake; (ii) personally witnessing death, serious injury, or the collapse of buildings; and (iii) not suffering any physical injury. Exclusion criteria included psychiatric comorbidity assessed using the Structured Clinical Interview for DSM-IV, a history of psychiatric or neurological disorders, recent medication that might affect the brain function, alcohol or drug abuse, and pregnancy. Each participant was assessed using the PTSD Checklist (PCL), a 17-item self-report measure of symptoms of PTSD (Weathers et al, 1994). This revealed a high degree of individual variability in symptoms of PTSD. Of the 121 participants included, 65 met the criteria for diagnosis of PTSD at the time of scanning.

\section{Resting-State Functional MRI Data Acquisition}

Each participant was investigated using resting-state functional MRI, a technique that provides information on spontaneous fluctuations in neuronal activity at rest. A total of 205 images sensitized to changes in BOLD signal levels were obtained from all participants using a three T MRI system (EXCITE, General Electric, Milwaukee, WI, USA) with a gradient-echo echo-planar imaging (EPI) sequence.
Table I Subject Socio-Demographic and Clinical Information (mean (SD))

\begin{tabular}{lc}
\hline Characteristic & $\begin{array}{c}\text { Earthquake } \\
\text { survivors }(\boldsymbol{n}=\mathbf{I} \mathbf{2} \mathbf{I})\end{array}$ \\
\hline Age (years) & $43.27(9.89)$ \\
Gender (M:F) & $40: 8 \mathrm{I}$ \\
Years of education (years) & $7.02(3.32)$ \\
PCL score & $38.45(\mathbf{I} 3.8 \mathrm{I})$ \\
\hline
\end{tabular}

Abbreviations: F, female; M, Male; PCL, PTSD Checklist; PTSD, post-traumatic stress disorder.

Sequence parameters were as follows: repetition time/echo time $(\mathrm{TR} / \mathrm{TE})=2000 / 30 \mathrm{~ms}$; flip angle $=90^{\circ} ; 30$ axial slice per volume; $5 \mathrm{~mm}$ slice thickness (no slice gap); matrix $=64$ $\times 64 ; \quad \mathrm{FOV}=240 \times 240 \mathrm{~mm}^{2} ;$ voxel size $=3.75 \times 3.75 \times 5$ $\mathrm{mm}^{3}$. Each functional run contained 205 image volumes, resulting in a total scanning time of $410 \mathrm{~s}$ for each participant. The first five scans were discarded before the preprocessing of the data to remove the impact of magnetization stabilization. All participants were instructed not to focus their thoughts on anything in particular and keep their eyes closed during the acquisition.

\section{Data Analysis}

Following image preprocessing using Statistical Parametric Mapping (SPM8; http://www.fil.ion.ucl.ac.uk/spm/) and the Resting State fMRI Data Analysis Toolkit (REST; http:// www.restfmri.net/forum), we performed the statistical analysis of the data using multivariate RVR (Tipping, 2001) as implemented in the Pattern Recognition for Neuroimaging Toolbox (PRoNTo; http://www.mlnl.cs.ucl.ac.uk/pronto/).

Image preprocessing. For each participant, EPI images were realigned to the first image and unwarped to correct for susceptibility-by-movement interaction using SPM8 software (http://www.fil.ion.ucl.ac.uk/spm/) running under Matlab (Mathworks, 2010 release). Examination of the movement parameters showed that there was no significant association between the mean $(r=0.008 ; P=0.931 ; n=121)$, or maximum $(r=-0.039 ; P=0.674 ; n=121)$, displacement, and clinical scores. All of the realigned images were spatially normalized to the Montreal Neurological Institute template and smoothed using an $8 \mathrm{~mm}$ full-width halfmaximum (FWHM) Gaussian kernel. Using the smoothed images, the average amplitude of low frequency fluctuation (ALFF), across the frequency band $0.01 \mathrm{~Hz}$ to $0.08 \mathrm{~Hz}$, was calculated within each voxel using REST software (http:// www.restfmri.net/forum) running under Matlab (Mathworks, 2010 release). The resulting spatial ALFF maps were then normalized with each voxel divided by the whole-brain ALFF mean, providing 'mALFF' spatial maps.

Univariate SPM analysis. We used a standard, univariate approach to investigate the relationship between clinical scores and the mean amplitude of spontaneous low frequency fluctuations during resting-state. A multiple 
regression model was used in SPM8 software to identify any voxels in the MALFF spatial maps that showed a significant association with clinical scores. Statistical inferences were made at $P<0.05$ (corrected for multiple comparisons using Family Wise Error (FWE)).

Multivariate RVR analysis. In addition, we examined the relationship between clinical scores and the mean amplitude of spontaneous low frequency fluctuations at rest using multivariate RVR as implemented in PRoNTo (http:// www.mlnl.cs.ucl.ac.uk/pronto/) running under Matlab (Mathworks, 2010 release). In brief, RVR is a sparse kernel learning multivariate regression method set in a fully probabilistic Bayesian framework. Under this framework, a zero-mean Gaussian prior is introduced over the model weights, governed by a set of hyperparameters-one for each weight. The most probable values for these hyperparameters are then iteratively estimated from the training data, with sparseness achieved due to the posterior distributions of many of the weights peaking sharply around zero; those training vectors associated with non-zero weights are referred to as 'relevance' vectors. The optimized posterior distribution over the weights can then be used to predict the target value (eg, PCL score) for a previously unseen input vector (eg, mALFF map) by computing the predictive distribution (for a more in-depth and detailed description see Tipping, 2001). In the current study, the input vectors (features) were mean centered using the training data, and an estimate for the model's generalizability obtained via leave-one-out cross validation, indexed using the Pearson correlation coefficient and mean squared error (MSE) between actual and predicted PCL score. The significance of both indices was estimated using a permutation test whereby the input-target data were randomly paired and the RVR rerun 1000 times. This created a distribution of correlation and MSE values reflecting the null hypothesis that the model did not exceed chance. The number of times the permuted value was greater than (or with respect to MSE values, less than), or equal to, the true value was then divided by 1000 providing an estimated $P$-value for both the correlation coefficient and observed MSE.

For ease of visualization, a table was also created using a $70 \%$ threshold for all successful RVR-derived weight maps, showing those regions with weight vector values in the upper, or lower, $30 \%$ of the absolute maximum and minimum weight vector values across all regions (Table 2). These values represent the relative contribution of each voxel to the regression function, in the context of every other voxel.

A priori mask. In order to take into account those regions most frequently implicated in PTSD by previous studies, ie, the bilateral prefrontal cortices, amygdalae, and hippocampi (Gilbertson et al, 2002; Pitman et al, 2012; Nardo et al, 2010; Robinson and Shergill, 2011; Shin et al, 2006; New et al, 2009; Lyoo et al, 2011; Morey et al, 2012; Weber et al, 2013), we also repeated both the univariate and multivariate analyses using an a priori mask comprising these regions (see SF1 in the Supplementary Data). This allowed us to examine whether the sensitivity of each analysis could be increased.

\section{RESULTS}

\section{Univariate SPM Analysis}

Whole brain analysis of the resting-state functional MRI data did not detect any regions that showed a significant association with clinical scores at $P<0.05$ (FWE corrected).

Using an a priori mask comprising regions implicated in PTSD, ie, the bilateral prefrontal cortices, amygdalae, and hippocampi (see SF1 in the Supplementary Data), this region of interest (ROI) analysis was also unable to detect any region(s) showing a significant association with clinical scores at $P<0.05$ (FWE corrected).

\section{Multivariate RVR Analysis}

The application of RVR to the whole-brain resting-state functional MRI data allowed quantitative prediction of clinical scores with statistically significant accuracy (correlation $=0.32 P$-value $=0.006$; mean sum of squares $=176.88 P$-value $=0.001$ ), see Figure 1 . Prediction was based on functional alterations across the whole brain, including in particular, frontal and parietal areas bilaterally in addition to cingulate, cerebellar, and subcortical regions, see Table 2 and Figure 2.

Using the same a priori mask as used in the univariate SPM analysis (see SF1 in the Supplementary Material), the multivariate analysis was also repeated. Accuracy of prediction was no longer significant (correlation $=-0.10 P$-value $=0.408 ;$ mean sum of squares $=247.03$ $P$-value $=0.647)$, suggesting that prediction of psychopathology was informed by regions outside of the bilateral frontolimbic network that is traditionally associated with PTSD.

\section{DISCUSSION}

Recent studies of psychiatric patients employing multivariate machine learning techniques have shown the potential of structural and functional neuroimaging for making inferences at the individual level (Orrù et al, 2012); however, these studies were limited to discrete categorical decisions such as whether an individual belongs to a patient or control group. For example, in a recent investigation, we showed that the application of a multivariate machine learning technique known as the SVM to structural MRI data, allows accurate distinction of traumaexposed individuals who do and do not meet criteria for PTSD (Gong et al, 2013). However, this study was based on a categorical distinction between patients and controls, and ignored individual variability in psychopathology within each subgroup. Here we have extended this earlier result by showing that the application of RVR to the whole-brain resting-state functional MRI data allows quantitative prediction of clinical scores with statistically significant accuracy. To our knowledge, this is the first evidence that neuroimaging techniques may inform the clinical assessment of psychiatric patients by allowing accurate and objective quantitative estimation of psychopathology.

The existing literature also suggests that psychological symptoms in PTSD are associated with dysfunction within a specific fronto-limbic network (Gilbertson et al, 
Table 2 Neuroanatomical Regions With a Weight Vector Score in the Top, or Bottom, 30\% of The Maximum Weight Vector Score across All Regions for the Resting-state Functional MRI-based RVR Used to Accurately Predict PCL Symptom Score. $w_{i}$, and MNI Coordinates Refer to the Peak Weight Vector Score in each Cluster

\begin{tabular}{|c|c|c|c|}
\hline Region & $\begin{array}{l}\text { Number } \\
\text { of voxels }\end{array}$ & $\begin{array}{c}\text { MNI } \\
\text { coordinate } \\
(x, y, z)\end{array}$ & $\left(\begin{array}{c}w_{i} \\
\left(\times 10^{-3}\right.\end{array}\right)$ \\
\hline
\end{tabular}

\begin{tabular}{|c|c|c|c|}
\hline \multicolumn{4}{|l|}{ Regions with positive $w_{i}$ scores } \\
\hline \multicolumn{4}{|l|}{ Frontal } \\
\hline Left superior frontal gyrus & 40 & $-12,34,60$ & 7.55 \\
\hline Left middle frontal gyrus & 25 & $-30,56,28$ & 6.46 \\
\hline $\begin{array}{l}\text { Left lateral fronto-orbital } \\
\text { gyrus }\end{array}$ & 17 & $-26,50,-18$ & 7.64 \\
\hline Right superior frontal gyrus & 16 & $22,4,72$ & 7.27 \\
\hline \multicolumn{4}{|l|}{ Parietal } \\
\hline \multirow[t]{2}{*}{ Left superior parietal lobule } & 75 & $-30,-80,48$ & 7.33 \\
\hline & 32 & $-36,-68,58$ & 7.64 \\
\hline \multirow[t]{2}{*}{ Left post central gyrus } & 56 & $-56,-28,48$ & 7.10 \\
\hline & । & $-24,-40,76$ & 5.99 \\
\hline Right angular gyrus & । & $56,-26,54$ & 5.75 \\
\hline
\end{tabular}

Regions with negative $w_{i}$ scores

Frontal

\begin{tabular}{|c|c|c|c|}
\hline \multirow[t]{2}{*}{ Right precentral gyrus } & 95 & $12,-20,78$ & -8.59 \\
\hline & 2 & $30,-22,72$ & -6.69 \\
\hline \multirow[t]{2}{*}{ Left medial frontal gyrus } & 79 & $-2,62,6$ & -7.93 \\
\hline & 9 & $-12,-14,78$ & -7.17 \\
\hline Left cingulate region & 40 & $-2,46,-14$ & -7.06 \\
\hline Right cingulate region & 20 & $0,34,-8$ & -7.81 \\
\hline Right middle frontal gyrus & 19 & $26,44,46$ & -7.27 \\
\hline Right superior frontal gyrus & । & $12,-8,78$ & -6.29 \\
\hline \multicolumn{4}{|l|}{ Occipital } \\
\hline Right cuneus & 24 & $6,-100,4$ & -7.77 \\
\hline Right superior occipital gyrus & 13 & $12,-84,48$ & -7.46 \\
\hline Right middle occipital gyrus & । & $42,-76,42$ & -6.52 \\
\hline \multicolumn{4}{|l|}{ Parietal } \\
\hline Right angular gyrus & 7 & $44,-56,58$ & -7.19 \\
\hline Left superior parietal lobule & 2 & $-30,-50,72$ & -6.07 \\
\hline \multicolumn{4}{|l|}{ Cerebellum } \\
\hline \multirow[t]{2}{*}{ Right cerebellum } & 9 & $28,-90,-24$ & -6.35 \\
\hline & 4 & $16,-92,-26$ & -6.35 \\
\hline \multicolumn{4}{|l|}{ Subcortical } \\
\hline Right uncus & 2 & $30,10,-32$ & -6.39 \\
\hline
\end{tabular}

Abbreviations: MNI, Montreal Neurological Institute; PCL, PTSD checklist; PTSD, post-traumatic stress disorder; RVR, relevance rector regression. $W_{i}$ : weight vector score indicating the relative contribution of each voxel to the regression function.

2002; Pitman et al, 2012; Nardo et al, 2010; Robinson and Shergill, 2011; Shin et al, 2006; New et al, 2009; Lyoo et al, 2011; Morey et al, 2012; Weber et al, 2013). Dysfunction in this network is thought to be explained by a combination of acquired signs of the disorder and pre-existing vulnerability factors (Sekiguchi et al, 2012). However, using a

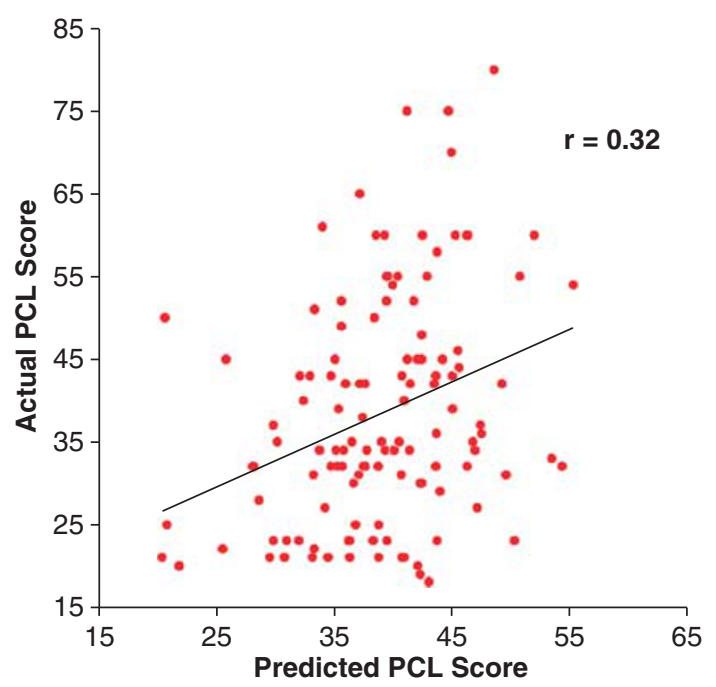

Figure I Scatter plot showing the predicted clinical score for each subject derived from their resting-state data using relevance vector regression (RVR), vs their actual clinical score.

whole-brain analysis approach, we found that a number of regions outside this fronto-limbic network traditionally associated with PTSD contributed to the accurate prediction of clinical scores. These included, for example, the left superior parietal lobule, right angular gyrus, right superior and middle occipital gyri, and right cerebellum and the right uncus (see Table 2 and Figure $1 \mathrm{~b}$ ). Furthermore, the accuracy of prediction was no longer significant when we repeated the analysis using an a priori mask comprising the bilateral prefrontal cortices, amygdalae, and hippocampi. Thus, although the majority of studies investigating PTSD have reported the bilateral prefrontal cortices, amygdalae, and/or hippocampi, our findings are consistent with the subset of studies that report structural and functional alterations in parietal and occipital regions also (Zhang et al, 2011; Chao et al, 2012; Tavanti et al, 2012; Liu et al, 2012; Eckart et al, 2011; Schuff et al, 2011). For instance Liu et al (2012) and Eckart et al (2011) reported a reduction of the right inferior parietal gyrus in terms of cortical thickness and volume, respectively, in participants with PTSD relative to healthy controls. Similarly, using arterial spin labeling MRI, Schuff et al (2011) noted an increased regional cerebral blood flow in the right parietal and superior temporal cortex of subjects with PTSD relative to those without. More recently, Tavanti et al (2012) reported a reduction in cortical gray matter volume in the frontal and occipital lobes of PTSD patients relative to healthy controls, which in turn appeared to correlate with clinical measures. Further consistent with this finding, Chao et al (2012) found that combat veterans diagnosed with PTSD had reduced left occipital lobe gray matter volume relative to those without the illness, which was negatively correlated with PTSD symptom severity. Our investigation therefore extends these studies by indicating that parietal and occipital regions provide critical information for successfully estimating PTSD symptomatology at individual level. This therefore supports the notion that regions outside the fronto-limbic network are critical for accurate prediction of psychopathology following exposure to trauma, and challenges the 


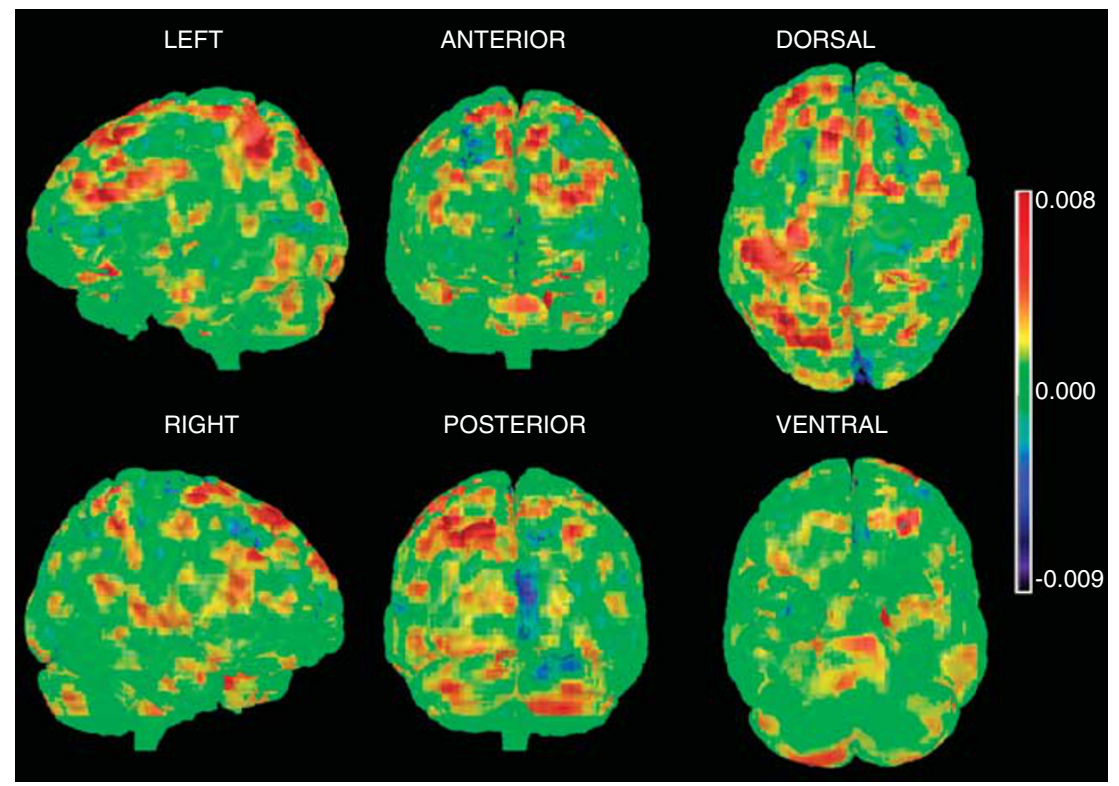

Figure 2 Multivariate map showing the weight of each voxel indicating its relative contribution to the regression function in the context of all other voxels (color bar in arbitrary units).

traditional view of PTSD as a disorder specific to frontal and limbic regions. In particular, the notable contribution of parietal and occipital regions to accurate prediction, suggests that the development of psychopathology following exposure to trauma may be associated with functional alterations in attentional and perceptual regions. This is consistent with cognitive models of PTSD that explain some of the main symptoms of the disorder in terms of altered attentional and perceptual processing (Ehlers and Clark, 2000). Similarly, the regions implicated here are in accordance with those areas often reported by studies investigating depersonalization disorder and social cognition, of which elements of both are frequently evident in those with PTSD (Phillips et al. 2001, Lemche et al. 2007; Lanius et al, 2011; Sierra and David 2011; Sugiura et al. 2013). However, it should be noted that in multivariate methods, an individual region may display high discriminative power due to two possible reasons: (i) a difference in signal intensity between individuals with low and high scores in that region; (ii) a difference in the correlation between that signal intensity of that region and other areas between individuals with low and high scores. Thus, the prefrontal, parietal, and occipital regions identified in the present investigation should be interpreted as a spatially distributed pattern rather than as independent areas.

In contrast, the univariate analysis of the resting-state functional MRI data, in which each voxel was considered as a spatially independent unit, did not detect any regions that showed a significant association with clinical scores. Critically, this finding remained unchanged when the $a$ priori mask of fronto-limbic regions as described above was used. Together, this provides support to the idea that multivariate methods such as RVR are more sensitive to the subtle and spatially diffuse alterations typically observed in psychiatric disorders, and therefore may be better suited to the development of a real-world clinical diagnostic tool, than standard mass-univariate techniques (Brammer, 2009).
Our finding that fluctuations in resting-state neural activity can accurately predict clinical symptomatology also builds on the growing number of studies which, using univariate techniques, reported an alteration of the restingstate neural networks in those with PTSD relative to healthy controls, which in many cases was in turn found to be correlated with clinical score (Zhou et al, 2012; Yin et al, 2012; Qin et al, 2012).

A limitation of the present study is that all participants were investigated after exposure to the earthquake using a cross-sectional design; thus, it was not possible to establish whether the observed variability in the brain function reflected plastic changes that occurred after the earthquake or pre-existing differences associated with individual variability in psychological vulnerability. In contrast, a recent investigation employing a longitudinal design was able to distinguish between morphological brain changes that reflect acquired signs of post-earthquake stress and pre-existing vulnerability at the group level (Sekiguchi et al, 2012). Another limitation is that all participants were free from psychiatric comorbidity and were not taking any medication that might affect the brain function. Although this had the advantage of minimizing the impact of these potential confounds on the results, it meant that our sample might not be fully representative of the population with PTSD. Further studies using a more representative sample including individuals with and without comorbidity would be required to better characterize the potential of restingstate functional MRI as a clinically useful diagnostic aid.

In conclusion, our results provide preliminary support to the development of resting-state functional MRI as a clinically useful diagnostic aid. Such aid could potentially be used in a clinical setting to inform the clinical evaluation of those difficult to categorize using traditional methods of clinical assessment alone (Pace and Heim, 2011; Lanius et al, 2007). In addition, it could potentially be employed in forensic evaluations of mental insanity by providing an 
objective means of reducing controversy and detecting malingering (Sartori et al, 2011). Nevertheless, it should be stressed that the eventual use of resting-state functional MRI in clinical practice would ultimately require a more comprehensive evaluation of clinical utility that takes several factors into account such as outcome impact, cost effectiveness, potential risks and side-effects, inconvenience, and ethical considerations.

\section{FUNDING AND DISCLOSURE}

The authors declare no conflict of interest.

\section{ACKNOWLEDGEMENTS}

This work was supported by grants from the National Natural Science Foundation of China awarded to Dr Qiyong Gong and Dr Andrea Mechelli (81220108013), Dr Qiyong Gong (81030027 and 81227002) and Prof Lingjiang Li (30830046 and 81171286). Dr Qiyong Gong also acknowledges the support from his CMB Distinguished Professorship Award (Award No. F510000/ G16916411) and the Program for Changjiang Scholars and Innovative Research Team in University (PCSIRT (IRT1272) of China. Dr Andrea Mechelli and Dr William Pettersson-Yeo acknowledge support from Medical Research Council Funding (grant ID 99859). Dr Nicolas Crossley is funded by a Wellcome Trust Clinical Research Training Fellowship (WT093907).

\section{REFERENCES}

Brammer M (2009). The role of neuroimaging in diagnosis and personalized medicine-current position and likely future directions. Dialogues Clin Neurosci 11: 389-396.

Chao LL, Lenoci M, Neylan TC (2012). Effects of post-traumatic stress disorder on occipital lobe function and structure. Neuroreport 23: 412-419.

Eckart C, Stoppel C, Kaufmann J, Tempelmann C, Hinrichs H, Elbert T et al (2011). Structural alterations in lateral prefrontal, parietal and posterior midline regions of men with chronic posttraumatic stress disorder. J Psychiatry Neurosci 36: 176-186.

Ehlers A, Clark DM (2000). A cognitive model of posttraumatic stress disorder. Behav Res Ther 38: 319-345.

Gilbertson MW, Shenton ME, Ciszewski A, Kasai K, Lasko NB, Orr SP et al (2002). Smaller hippocampal volume predicts pathologic vulnerability to psychological trauma. Nat Neurosci 5: 1242-1247.

Gong Q, Li L, Tognin S, Wu Q, Pettersson-Yeo W, Lui S et al (2013). Using structural neuroanatomy to identify trauma survivors with and without post-traumatic stress disorder at the individual level. Psychol Med 3: 1-9.

Lanius RA, Bluhm RL, Frewen PA (2011). How understanding the neurobiology of complex post-traumatic stress disorder can inform clinical practice: a social cognitive and affective neuroscience approach. Acta Psychiatr Scand 124: 331-348.

Lanius RA, Frewen PA, Girotti M, Neufeld RW, Stevens TK, Densmore M (2007). Neural correlates of trauma script-imagery in posttraumatic stress disorder with and without comorbid major depression: a functional MRI investigation. Psychiatry Res 155: 45-56.

Lemche E, Surguladze SA, Giampietro VP, Anilkumar A, Brammer MJ, Sierra M et al (2007). Limbic and prefrontal responses to facial emotion expressions in depersonalization. Neuroreport 18: 473-477.
Liu Y, Li YJ, Luo EP, Lu HB, Yin H (2012). Cortical thinning in patients with recent onset post-traumatic stress disorder after a single prolonged trauma exposure. PLoS One 7: e39025.

Lyoo IK, Kim JE, Yoon SJ, Hwang J, Bae S, Kim DJ (2011). The neurobiological role of the dorsolateral prefrontal cortex in recovery from trauma. Longitudinal brain imaging study among survivors of the South Korean subway disaster. Arch Gen Psychiatry 68: 701-713.

Morey RA, Gold AL, LaBar KS, Beall SK, Brown VM, Haswell CC et al Mid-Atlantic MIRECC Workgroup (2012). Amygdala volume changes in posttraumatic stress disorder in a large case-controlled veterans group. Arch Gen Psychiatry 69: 1169-1178.

Nardo D, Hogberg G, Looi JC, Larsson S, Hallstrom T, Pagani M (2010). Gray matter density in limbic and paralimbic cortices is associated with trauma load and EMDR outcome in PTSD patients. J Psychiatr Res 44: 477-485.

New AS, Fan J, Murrough JW, Liu X, Liebman RE, Guise KG et al (2009). A functional magnetic resonance imaging study of deliberate emotion regulation in resilience and posttraumatic stress disorder. Biol Psychiatry 66: 656-664.

Orrù G, Pettersson-Yeo W, Marquand AF, Sartori G, Mechelli A (2012). Using Support Vector Machine to identify imaging biomarkers of neurological and psychiatric disease: a critical review. Neurosci Biobehav Rev 36: 1140-1152.

Pace TW, Heim CM (2011). A short review on the psychoneuroimmunology of posttraumatic stress disorder: from risk factors to medical comorbidities. Brain Behav Immun 25: 6-13.

Phillips M L, Medford N, Senior C, Bullmore E T, Suckling J, Brammer M J et al (2001). Depersonalization disorder: thinking without feeling. Psychiatry Res 108: 145-160.

Pitman RK, Rasmusson AM, Koenen KC, Shin LM, Orr SP, Gilbertson MW et al (2012). Biological studies of post-traumatic stress disorder. Nat Rev Neurosci 13: 769-787.

Qin LD, Wang Z, Sun YW, Wan JQ, Su SS, Zhou Y et al (2012). A preliminary study of alterations in default network connectivity in post-traumatic stress disorder patients following recent trauma. Brain Res 1484: 50-56.

Robinson BL, Shergill SS (2011). Imaging in posttraumatic stress disorder. Curr Opin Psychiatry 24: 29-33.

Rosen GM, Taylor S (2007). Pseudo-PTSD. J Anxiety Disord 21: 201-210.

Sartori G, Pellegrini S, Mechelli A (2011). Forensic neurosciences: from basic research to applications and pitfalls. Curr Opin Neurol 24: 371-377.

Savitz JB, Rauch SL, Drevets WC (2013). Clinical application of brain imaging for the diagnosis of mood disorders: the current state of play. Mol Psychiatry 18: 528-539.

Schuff N, Zhang Y, Zhan W, Lenoci M, Ching C, Boreta L et al (2011). Patterns of altered cortical perfusion and diminished subcortical integrity in posttraumatic stress disorder: an MRI study. Neuroimage 54(Suppl 1): S62-S68.

Sekiguchi A, Sugiura M, Taki Y, Kotozaki Y, Nouchi R, Takeuchi H et al (2012). Brain structural changes as vulnerability factors and acquired signs of post-earthquake stress. Mol Psychiatry 18: 618-623.

Shin LM, Rauch SL, Pitman RK (2006). Amygdala, medial prefrontal cortex, and hippocampal function in PTSD. Ann N Y Acad Sci 1071: 67-79.

Sierra M, David AS (2011). Depersonalization: a selective impairment of self-awareness. Conscious Cogn 20: 99-108.

Sugiura M, Yomogida Y, Mano Y, Sassa Y, Kambara T, Sekiguchi A et al (2013). From social-signal detection to higher social cognition: an fMRI approach. Soc Cogn Affect Neurosci (Epub ahead of print).

Tavanti M, Battaglini M, Borgogni F, Bossini L, Calossi S, Marino D et al (2012). Evidence of diffuse damage in frontal and occipital 
cortex in the brain of patients with post-traumatic stress disorder. Neurol Sci 33: 59-68.

Tipping ME (2001). Sparse Bayesian learning and the relevance vector machine. J Mach Learn Res 1: 211-244.

Weathers FW, Litz BT, Huska JA, Keane TM (1994). PTSD Checklist-Civilian Version. Boston, National Center for PTSD, Behavioural Science Division: Boston, MA, USA.

Weber M, Killgore WD, Rosso IM, Britton JC, Schwab ZJ, Weiner MR et al (2013). Voxel-based morphometric gray matter correlates of posttraumatic stress disorder. J Anxiety Disord 27: 413-419.

Yehuda R, Flory JD (2007). Differentiating biological correlates of risk, PTSD, and resilience following trauma exposure. J Trauma Stress 20: 435-447.

Yin Y, Jin C, Eyler LT, Jin H, Hu X, Duan L et al (2012). Altered regional homogeneity in post-traumatic stress disorder: a resting-state functional magnetic resonance imaging study. Neurosci Bull 28: 541-549.

Zhang J, Tan Q, Yin H, Zhang X, Huan Y, Tang L et al (2011). Decreased gray matter volume in the left hippocampus and bilateral calcarine cortex in coal mine flood disaster survivors with recent onset PTSD. Psychiatry Res 192: 84-90.

Zhou Y, Wang Z, Qin LD, Wan JQ, Sun YW, Su SS et al (2012). Early altered resting-state functional connectivity predicts the severity of post-traumatic stress disorder symptoms in acutely traumatized subjects. PLoS One 7: e46833.

(c) (i) (-) $\odot$ This work is licensed under a Creative Commons Attribution-NonCommercial-NoDerivs 3.0 Unported License. To view a copy of this license, visit http:// creativecommons.org/licenses/by-nc-nd/3.0/

Supplementary Information accompanies the paper on the Neuropsychopharmacology website (http://www.nature.com/npp) 